\title{
Optimum Copper-Palladium Catalyst from a Combinatorial Library for Sensitive Non-Enzymatic Glucose Sensors
}

\author{
Isabella Pötzelberger ${ }^{1}$. Cezarina Cela Mardare ${ }^{2} \cdot$ Lisa Maria Uiberlacker $^{3}$. \\ Sabine Hild ${ }^{3}$ • Achim Walter Hassel ${ }^{1,2}$
}

Published online: 23 November 2017

(C) The Author(s) 2017. This article is an open access publication

\begin{abstract}
The optimum activity for glucose electrocatalytic oxidation was found by screening along a large spread cosputtered combinatorial copper-palladium library (2.6 at.\% to 39.2 at.\% Pd) in neutral media using flow-type scanning droplet cell microscopy (FT-SDCM). The elemental composition and the surface topography were characterized along the compositional spread using energy dispersive X-ray spectroscopy (EDX), as well as atomic force microscopy (AFM). The study proves that the entire range of alloys can be implemented in glucose detection. The highest catalytic effect was obtained at a Pd content of 8.2 at.\% $\left(E_{\mathrm{SHE}}=0.58 \mathrm{~V}\right)$ with a current density value of $7.33 \mathrm{~mA} \mathrm{~cm}{ }^{-2}$. The suitability for being used as quantitative and qualitative glucose sensor was demonstrated. The calibration performed in phosphate buffer solution containing different amounts of glucose revealed two linear regions with different sensitivities towards the quantitative glucose detection. The highest sensitivity was determined in the range of $0-25 \mathrm{mM}$ glucose, which is indicated by an increase of $81.2 \mu \mathrm{A} \mathrm{cm}^{-2} \mathrm{mM}^{-1}$, normalized to the stepwise increase of $1 \mathrm{mM}$ glucose. Also, a good longterm stability, reproducibility (relative standard deviation $\sim 5 \%$ ), as well as the selective sensitivity to glucose oxidation were demonstrated by performing measurements in the
\end{abstract}

Achim Walter Hassel

achimwalter.hassel@jku.at

1 Institute for Chemical Technology of Inorganic Materials, Johannes Kepler University Linz, Altenberger Str. 69, 4040 Linz, Austria

2 Christian Doppler Laboratory for Combinatorial Oxide Chemistry, Institute for Chemical Technology of Inorganic Materials, Johannes Kepler University Linz, Altenberger Str. 69, 4040 Linz, Austria

3 Institute of Polymer Science, Johannes Kepler University Linz, Altenberger Str. 69, 4040 Linz, Austria presence of other compounds found in blood (e.g., ascorbic acid and uric acid).

Keywords Copper-palladium $\cdot$ Co-sputtered combinatorial material library · Thin film · Glucose .

Electrocatalytic oxidation

\section{Introduction}

Various technological studies on the development and analysis of combinatorial materials have attracted an increased attention in the last decade. Previous studies have emphasized the benefits of combinatorial approaches. Combining various methods in the field of electrochemistry and analytics allows multiple experiments which provide a considerable number of data in a relatively short time [1-3]. New materials such as binary, ternary, or even $n$-component materials can be fabricated in a fast way and in a one-step experiment. The fabrication methods may be different, such as co-sputtering [4, 5], thermal co-evaporation [6, 7], as well as electrodeposition [8] of thin film combinatorial libraries. One characteristic of these thin film material libraries is the change of the composition along the entire sample length (or length and width for ternary systems) as a function of the position on the substrate. Consequently, a change in the material properties within the material library is obtained. Recent reports revealed that screening along a $\mathrm{Cu}-\mathrm{Pd}$ compositional spread led to the determination of an optimum composition with augmented electrocatalytic activity towards glucose oxidation [9]. In the last years, the development of electrode materials for fast detection of biological substances such as amino acids, enzymes, as 
well as the concentration of the blood glucose level has received an increased interest [10]. These sensing materials have many promising application fields such as food industry, glucose-air fuel cells, in clinical detection and for environmental monitoring [11-13]. The development of suitable electrochemical glucose sensors for an accurate detection of glucose concentration has attracted special attention due to their simplicity, high sensitivity, reliability, good selectivity, fast response, low-cost fabrication, compatibility to miniaturization, and low detection limit. For persons with diabetes mellitus disease, the development of new materials or even the improvement of such sensing materials for monitoring the concentration of blood glucose is of high importance. Previous studies describe the development of enzymatic glucose sensors [10]. However, the stability of the enzymes depends to a large extent on temperature, humidity, as well as the $\mathrm{pH}$, and in special cases also on radiation during an overseas flight, and disadvantages such as the complicated immobilization process or operational parameters require that alternative materials are found. Consequently, non-enzymatic glucose sensors have attracted increased attention. Various metals such as $\mathrm{Au}[14,15], \mathrm{Pt}[16,17]$, and Pd [18, 19] are used for the electrocatalytic oxidation of glucose. Clear advantages such as higher catalytic activity, lower costs, and lower poisoning effects on the catalysts' surface make pure Pd particularly suitable for being used as electrocatalyst as compared to pure Pt. Nevertheless, disadvantages such as high material costs and poisoning effects on the catalysts surface triggered the search of alternative materials. Alloying Pd with a nonnoble metal lowers the material cost tremendously [9]. Cu and its alloys showed good corrosion resistance and electrocatalytic behavior towards glucose oxidation [9, 20-22].

Therefore, in this work, the promising properties of pure $\mathrm{Cu}$ and $\mathrm{Pd}$ were combined for the first time to investigate the electrocatalytic oxidation of glucose on a combinatorial thin film material library. A co-sputtered $\mathrm{Cu}-\mathrm{Pd}$ thin film library with a continuous compositional spread was used for electrocatalytic oxidation of glucose using a flow-type scanning droplet cell microscope (FT-SDCM).

\section{Experimental Details}

\section{Fabrication of the Cu-Pd Thin Film Combinatorial Library}

The copper-palladium (Cu-Pd) thin film material library was deposited by co-sputtering in a multi sputter gun system $(\mathrm{CODO})$ on glass substrates $(26 \mathrm{~mm} \times 76 \mathrm{~mm})$ which were previously coated with a thin $\mathrm{Cr}$ layer $(20 \mathrm{~nm})$ necessary as adhesion promoter. Prior to the metals $(\mathrm{Cr}, \mathrm{Cu}$, and $\mathrm{Pd})$ deposition, the base pressure in the sputtering chamber was approximately $10^{-8} \mathrm{hPa}$. The depositions were carried out at a pressure of $0.8 \mathrm{~Pa}$, in Ar. At first, the glass substrates were coated with the thin $\mathrm{Cr}$ layer. In order to obtain a uniform $\mathrm{Cr}$ thickness, the substrates have been rotated during the sputtering process. Afterwards, without breaking the vacuum, the co-deposition of $\mathrm{Cu}$ and $\mathrm{Pd}$ has been performed at room temperature and without substrates rotation. The target arrangement is in confocal geometry with $180^{\circ}$ between them around the normal of the substrate and $90^{\circ}$ against each other in the plane of incident beam. Using this geometry, a composition gradient along the substrates is achieved. In order to obtain the desired compositional spread, three different independent processes had to be performed at different sputtering powers on the $\mathrm{Cu}$ and Pd cathodes. The deposition time was $45 \mathrm{~min}$ for all samples. Sample I has been deposited using $6 \mathrm{~W}$ sputtering power for $\mathrm{Pd}$ and $100 \mathrm{~W}$ for $\mathrm{Cu}$, sample II with $11 \mathrm{~W}$ for $\mathrm{Pd}$ and $80 \mathrm{~W}$ for $\mathrm{Cu}$, and sample III with $25 \mathrm{~W}$ for $\mathrm{Pd}$ and $74 \mathrm{~W}$ for $\mathrm{Cu}$. The aforementioned necessary power values were calculated using a self-developed software based on a mixed matter theory in order to obtain the desired compositional spreads. The target diameter was 2 in. $(50.8 \mathrm{~mm})$.

The elemental composition was characterized using energy dispersive X-ray spectroscopy by screening along the entire compositional spread (INCA X-Sight Oxford Instruments). Surface roughness was determined by means of scanning force microscopy (Asylum Research MFP-3D Stand Alone AFM). On each area, three $1 \times 1 \mu^{2}$ scans were performed to calculate the root mean square $\left(R_{\mathrm{q}}\right)$ values. All AFM measurements were carried out in intermittent contact mode (set point $65 \%$ ) in air.

\section{Flow-Type Scanning Droplet Cell Microscopy}

For all electrochemical investigations, a flow-type scanning droplet cell (FT-SDCM) was used. The electrochemical cell consists of a 3D-printed polymeric block, which was designed for a three-electrode configuration. At the tip of the FT-SDCM, a small droplet of the electrolyte was formed and brought in contact with the investigated surface. For a proper sealing, a sealing ring (polymeric rubber, $0.5 \mathrm{~mm}$ thick) was glued at the tip of the FT-SDCM exactly matching the tip opening. Detailed information about the construction and operation of the FTSDCM can be found elsewhere [23]. An Au wire serves as counter electrode and an $\mathrm{AuHg} / \mathrm{Hg}_{2}\left(\mathrm{CH}_{3} \mathrm{COO}\right)_{2} / \mathrm{NaCH}_{3} \mathrm{COO}$ was fabricated as reference electrode. Therefore, a high purity Au wire (99.999\%) was cleaned with acetone and ethanol in an ultrasonic bath for $5 \mathrm{~min}$. The deposition of the mercury was done by polarizing the Au wire to $0.2 \mathrm{~V}$ (SHE) for $180 \mathrm{~s}$ in a $0.1 \mathrm{M} \mathrm{Hg}_{2}\left(\mathrm{NO}_{3}\right)_{2}$ electrolyte solution. Then, the amalgamated wire was rinsed with distilled water. For the deposition of $\mathrm{Hg}_{2}\left(\mathrm{CH}_{3} \mathrm{COO}\right)_{2}$, a $1 \mathrm{M} \mathrm{NaCH}_{3} \mathrm{COO}$ solution was prepared. The deposition of $\mathrm{Hg}_{2}\left(\mathrm{CH}_{3} \mathrm{COO}\right)_{2}$ was done by polarizing to $0.58 \mathrm{~V}$ (SHE) for $600 \mathrm{~s}$. To improve the mechanical stability, the wire was inserted into a Teflon tube and filled with a hot 
mixture of $1 \mathrm{M} \mathrm{NaCH}_{3} \mathrm{COO}$ and 4 wt.\% agar. After cooling and solidification, the fabricated reference electrode was stored in $1 \mathrm{M} \mathrm{NaCH}_{3} \mathrm{COO}$ electrolyte solution [24]. Counter and reference electrodes were inserted into the FT-SDCM. A selfdeveloped peristaltic pump was used for filling and flushing the volume of the electrochemical cell through an electrolyte inlet/outlet. All electrochemical experiments were performed in contact mode by pressing the cell with a defined force of $2 \mathrm{~N}$ against the analyzed surface in order to guarantee a precise delimitation of the wetted area. Due to the non-uniform elastic deformation of the sealing ring, the examined surface was determined by local anodization of a valve metal [25]. For this purpose, a Ti plate was anodized using an acetate buffer solution with $\mathrm{pH}=6.0$. The areas of the obtained colored $\mathrm{TiO}_{2}$ spots were measured using optical microscopy [26]. The wetted area on the working electrode was determined to be $0.984 \mathrm{~mm}^{2}$. For the electrochemical study, chemicals of analytical grade were used with no further purifications. All electrochemical investigations were performed using a CompactStat Potentiostat (Ivium Technology, The Netherlands).

\section{Results and Discussion}

The EDX characterization performed on the library (Fig. 1) revealed an overall compositional ranging from approximately 2.5 at.\% $\mathrm{Pd}$ to 39 at.\% $\mathrm{Pd}$ (complementary $\mathrm{Cu}$ ). The sputtering powers and consequently the achieved spreads have been adjusted in such way that between two adjacent samples, there is a compositional overlap on a significant length from the sample's edge $(\approx 25 \mathrm{~mm})$. This overlap was especially tuned in order to observe if there is an influence of the sample preparation conditions and/or edge effects on the electrocatalytic properties under investigation. The samples have been characterized in several points by X-ray diffraction for crystallographic information, and only the presence of a $\mathrm{Cu}$ phase with face-center cubic structure was found. Detailed analysis of the diffractograms, with the lattice parameters and crystallite size calculations, as well as scanning electron microscopy of the surface as a function of Pd content for a similar library, was reported somewhere else [9].

AFM images of the surface acquired in selected points along the library are presented in Fig. 2. As indicated by the surface topography, there is no significant change of the grains' morphology with increasing Pd concentration. The only difference is in the grains' maximum height, which, as a general trend, is increasing with increasing Pd concentration from approximately $20 \mathrm{~nm}$ for low Pd concentration to $50 \mathrm{~nm}$ for higher Pd content. However, along each sample, there are variations in the maximum height with the values not showing a clear dependency on the Pd concentration increase. The same type of dependency is found when analyzing the surface roughness (expressed by root mean square of roughness$R_{\mathrm{q}}$ ) as shown in Fig. 3. For each Pd concentration, the $R_{\mathrm{q}}$ values were obtained from measurements performed in three different areas in order to obtain statistical information about the surface. The standard deviation values are shown as error bars in Fig. 3.

The Pd concentrations where the AFM measurements have been performed were chosen in such a way that there was an overlap in the range of approximately 7 to 10 at.\% Pd between sample I and II. Following the trend of the grains' maximum height from the 3D images of the surface (Fig. 2), also the roughness of sample I is decreasing from approximately $3.5 \mathrm{~nm}$ for 2.9 at.\% Pd to $2.5 \mathrm{~nm}$ for 7.3 at.\% Pd, followed by a sudden increase to $3.3 \mathrm{~nm}$ for 8.4 at. $\%$, and then a drop in $R_{\mathrm{q}}$ for 9.7 at.\% Pd. The same non-linear variation of $R_{\mathrm{q}}$ as a function of Pd concentration increase is seen in sample II, with a sudden increase of $R_{\mathrm{q}}$ for 7.9 at.\% Pd, then followed by a drop in the $R_{\mathrm{q}}$ value. Interestingly, exactly in the same compositional region between 7.5 and 9 at.\% Pd, there is an extremely large standard deviation in the roughness values on both sample I and II, indicating a very non-uniform surface roughness local distribution. For higher Pd concentrations, the $R_{\mathrm{q}}$ values are steadily increasing, even though the standard deviation values are high as well. This fact can be related to

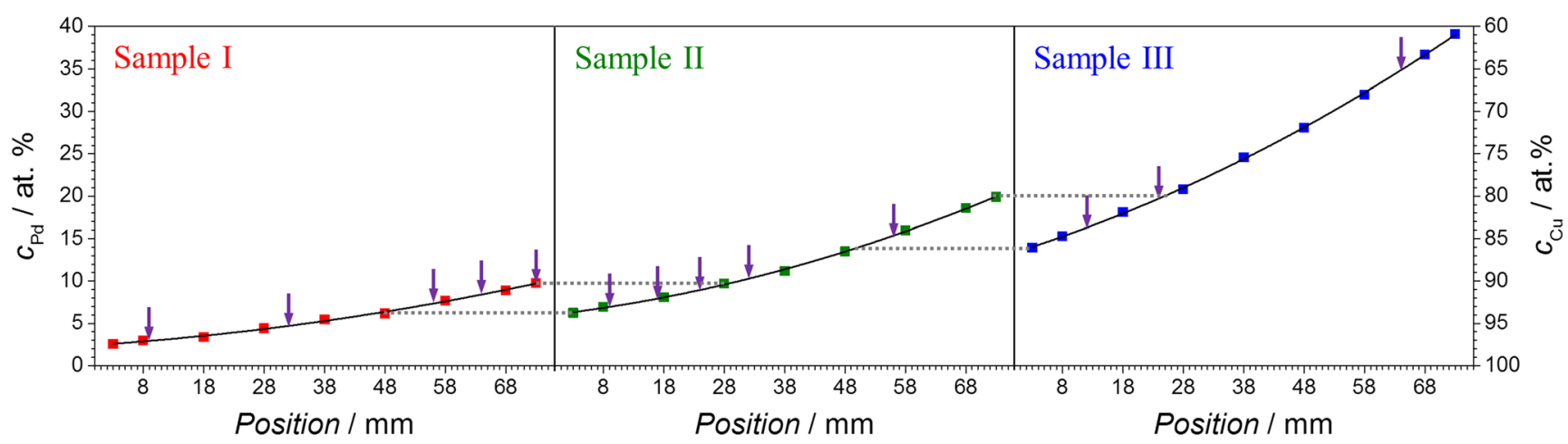

Fig. 1 EDX composition along the three samples showing the Pd concentration (left $y$-axis) and $\mathrm{Cu}$ concentration (right $y$-axis) as a function of position on each substrate. The dotted horizontal lines indicate

the compositional overlap between two adjacent samples. The violet arrows indicate the positions where AFM measurements were performed 


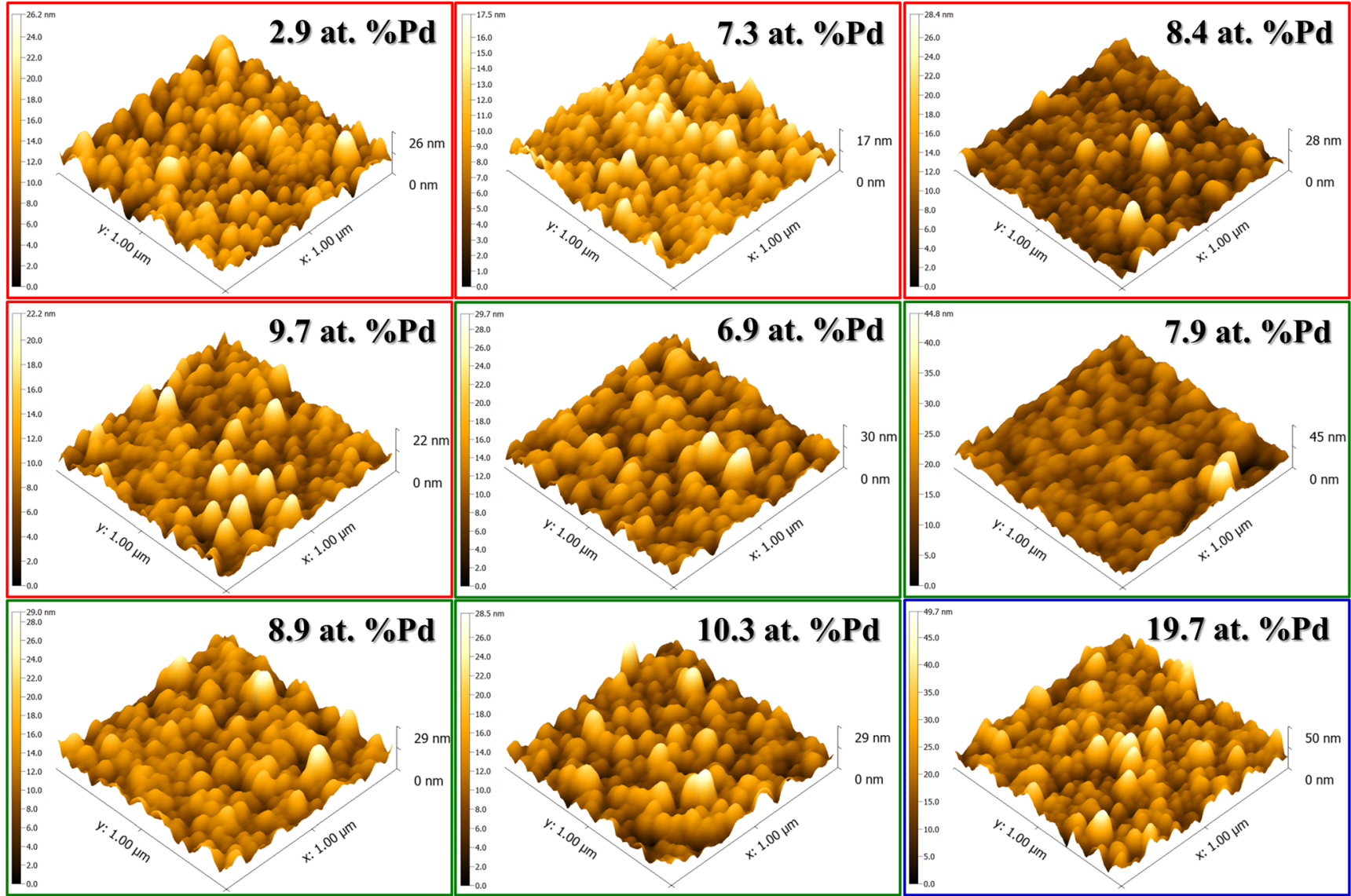

Fig. 2 AFM images of the surface in selected points along the library. The Pd concentration is indicated on each image. The color of the frame indicates the sample (red for sample I, green for sample II, and blue for sample III) on which the measurement was performed

Fig. 3 Root mean square values of roughness as a function of Pd concentration measured in selected points along the library. The red, green, and blue colors indicate the sample. The dotted lines represent eye guidance only

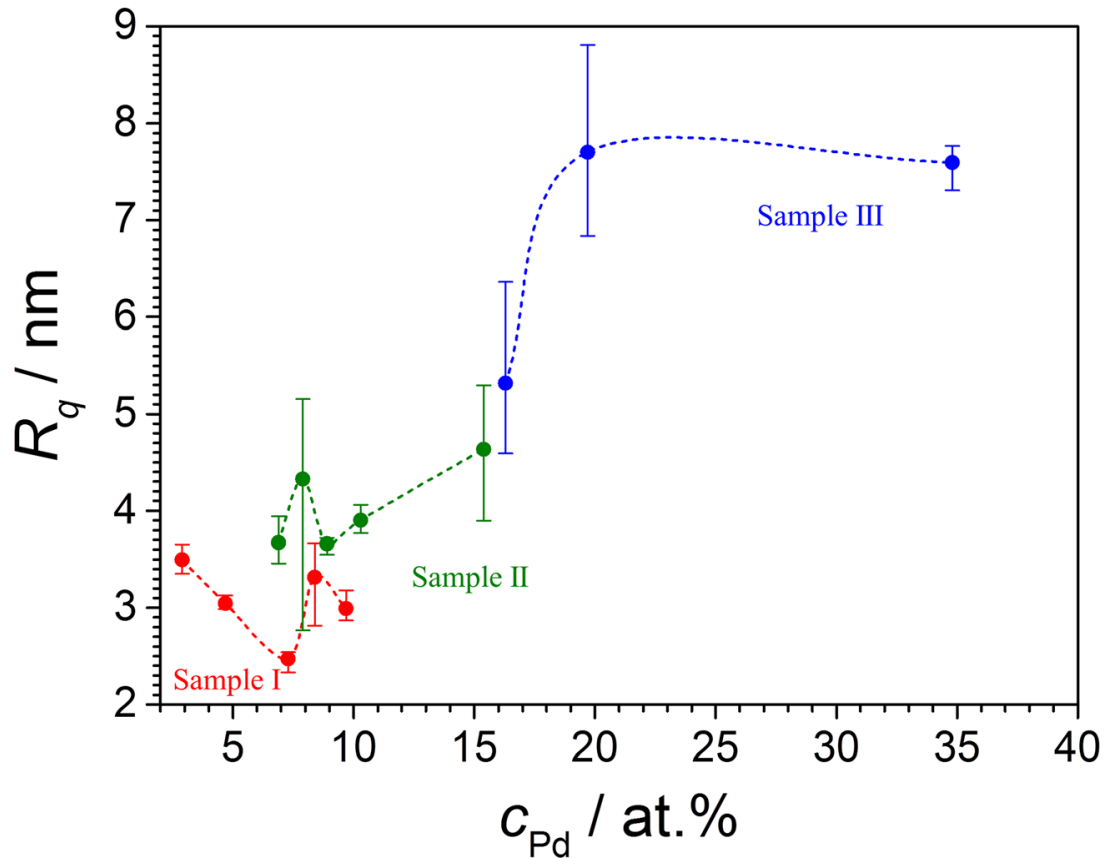


the coalescence of small crystallite and the subsequent formation of a few larger grains for concentrations higher than approximately 10 at.\% Pd [9], as also observed in the images from Fig. 2. The presence of these higher, isolated grains lead most probably to an increase in roughness, and simultaneously to very different $R_{\mathrm{q}}$ values for the same composition.

\section{Electrocatalytic Oxidation of Glucose}

The electrocatalytic oxidation of glucose was investigated by screening along $\mathrm{Cu}$-Pd thin film combinatorial library in phosphate buffer solution (PBS) at $\mathrm{pH}$ 7.4. Cyclic voltammetric measurements in a maximum range of -1.00 to $1.30 \mathrm{~V}$ vs. SHE with a potential increase rate of $0.02 \mathrm{~V} \mathrm{~s}^{-1}$ were performed. As reference, the obtained cyclic voltammograms (CV) for pure $\mathrm{Cu}$ and $\mathrm{Pd}$ with and without the addition of glucose are presented in Fig. 4. Part (a) of Fig. 4 shows the results of the measurements on a pure $\mathrm{Cu}$ thin film in the absence of glucose (dotted orange line). In the anodic scan
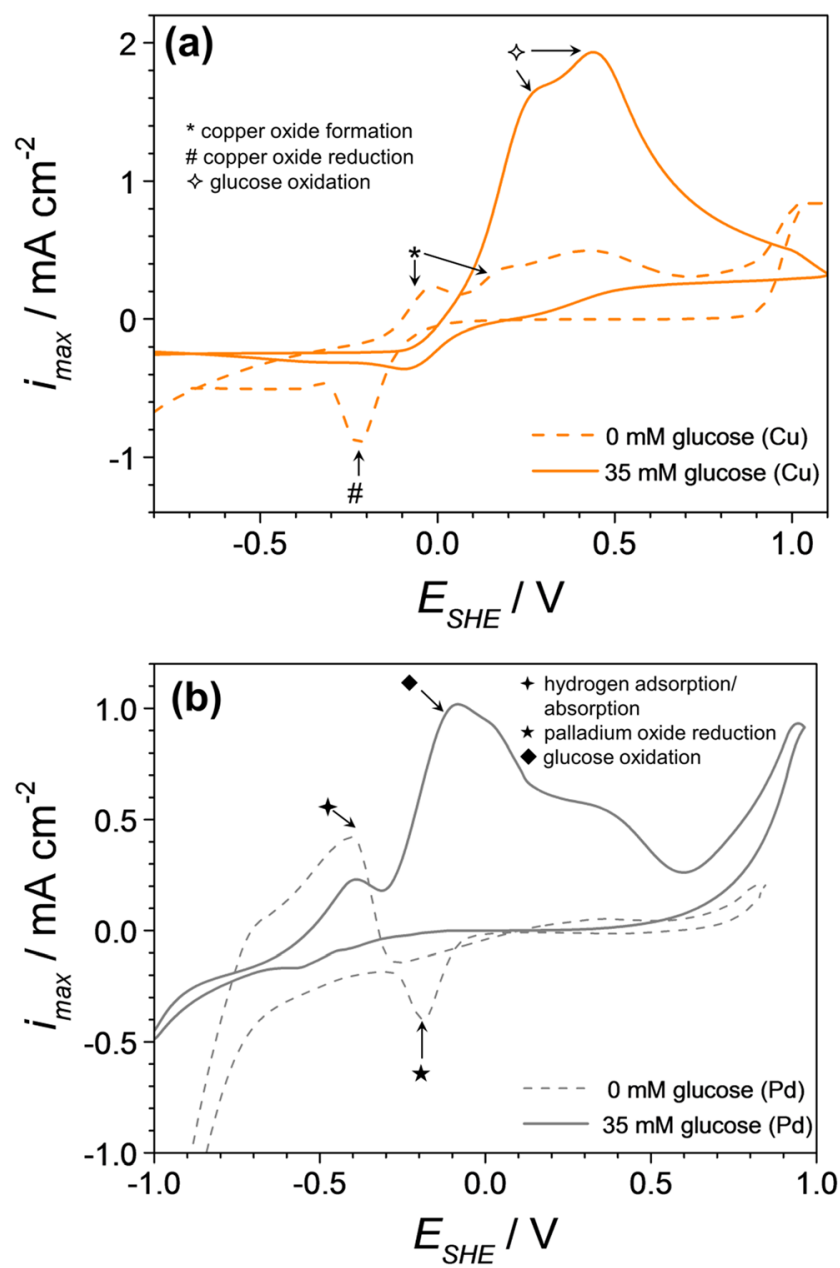

Fig. $4 \mathrm{CV}$ of pure $\mathrm{Cu}(\mathbf{a})$ and $\mathrm{Pd}(\mathbf{b})$ thin films with (continuous line) and without (dotted line) $35 \mathrm{mM}$ glucose direction, two well-defined anodic peaks are visible, which can be attributed to the $\mathrm{Cu}^{+}\left(-0.01 \mathrm{~V}\right.$ vs. SHE) and $\mathrm{Cu}^{2+}$ $(0.17 \mathrm{~V}$ vs. SHE) oxide formation [27]. In the cathodic scan direction, the observed peak at $-0.21 \mathrm{~V}$ (SHE) can be attributed to the $\mathrm{Cu}$ oxide reduction, which is in good agreement with previous studies [28]. In the presence of glucose $(35 \mathrm{mM})$ in the electrolyte solution, two well-defined anodic peaks at 0.26 and $0.45 \mathrm{~V}$ (SHE) were obtained (orange line). At a potential of $0 \mathrm{~V}$, the oxidation process started and is inhibited by the electrode oxidation, which is indicated by a sudden drop of the current density to the base current of the measurement without the addition of glucose.

Figure 5 shows the schematic illustration of the electrocatalytic oxidation of glucose that may proceed on the electrode surface. Generally, the glucose adsorbs on the metal surface during the oxidation process [29]. Due to the presence of $d$ electrons in the metallic electrode material, a favorable bond is formed between the adsorbed glucose molecule and the substrate. A change in the oxidation state of the sensing material can cause a change in the bonding strength and as a result, it allows the desorption of the oxidized species from the catalysts' surface [30, 31]. Fresh Cu-Pd surface is exposed and the glucose molecule can adsorb to the catalysts' surface from the electrolyte solution allowing the oxidation reaction to proceed.

Figure 4(b) presents the result of the cyclic voltammetric measurements of pure Pd in the absence (dotted gray line) and presence (gray line) of $35 \mathrm{mM}$ of glucose. From the measurements without the addition of glucose, a well-defined anodic peak was observed at a potential of $-0.40 \mathrm{~V}$ (SHE) due to the adsorption and absorption of hydrogen [32, 33]. At higher potentials $(>0.4 \mathrm{~V})$ in the anodic scan direction, $\mathrm{PdO}$ is formed [34]. At $-0.17 \mathrm{~V}$ (SHE), a cathodic peak was obtained indicating the $\mathrm{PdO}$ reduction $[34,35]$. After the addition of $35 \mathrm{mM}$ glucose to the electrolyte solution, an anodic peak at $-0.38 \mathrm{~V}$ (SHE) was obtained. Comparing the CV before and after the glucose addition, this anodic peak was still visible, but the current density was reduced by half as compared to the peak in the absence of glucose. The decrease of the current density value in the hydrogen adsorption region occurs due to the adsorption of glucose molecules on the electrode surface [31]. At $-0.08 \mathrm{~V}$ (SHE), a very strong increase in the current density value in the range from -0.3 to $0.14 \mathrm{~V}$ vs. SHE was observed, which corresponds to the glucose oxidation. The glucose molecule adsorbs on the electrode surface, and the organic species can be oxidized to gluconic acid due to reaction (1). The oxidation process continues and it is inhibited by the Pd oxide formation on the catalysts surface, which seems to be inactive for the glucose oxidation.

The electrocatalytic oxidation of glucose was also investigated on the $\mathrm{Cu}$-Pd thin film combinatorial library in a PBS having the $\mathrm{pH}=7.4$. Therefore, cyclic voltammograms were recorded in a maximum potential range from $-1.0 \mathrm{~V}$ (SHE) to $1.38 \mathrm{~V}$ (SHE) at various $\mathrm{Pd}$ concentrations along the entire 
Fig. 5 Schematic illustration of the reaction mechanism for the electrocatalytic glucose oxidation adapted from [29]

\section{Oxidation}

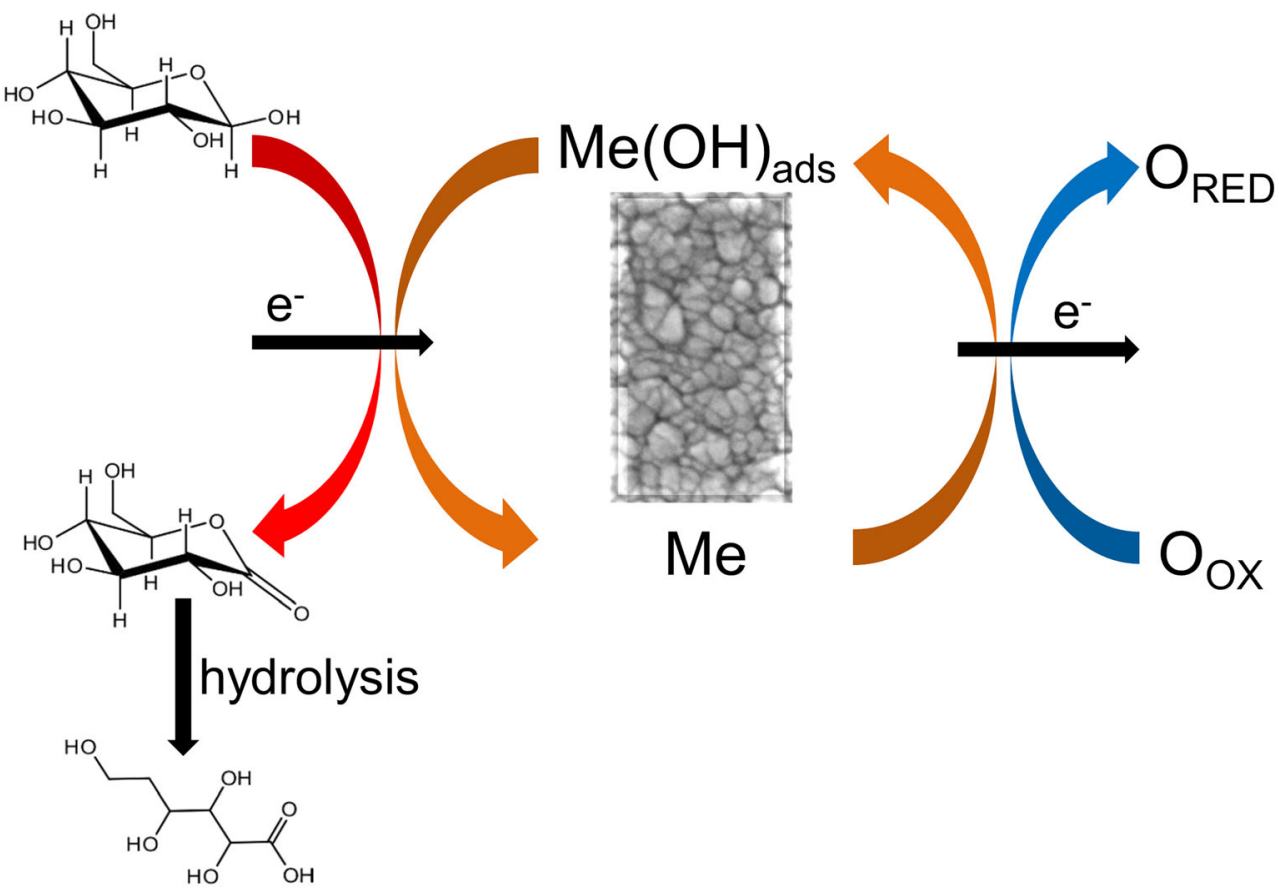

compositional spread with a rate of potential increase of $0.020 \mathrm{~V} \mathrm{~s}^{-1}$. The first cycles at selected Pd concentrations are presented in Fig. 6. As reference, the cyclic voltammograms which were measured without the addition of glucose are also presented and they show an identical behavior (dotted lines in Fig. 6). In the positive scan direction, an anodic peak was observed at $-0.06 \mathrm{~V}$ (SHE) for $\mathrm{Cu}-2.7$ at.\% Pd thin film alloy. The obtained peak can be attributed to the electrocatalytic glucose oxidation when compared to the measurement performed in the absence of glucose. Even the addition of a small amount of Pd has a large influence on the measured CV, which resulted in the shift of glucose oxidation potential from 0.26 to $-0.06 \mathrm{~V}$ vs. SHE. The oxidation process started at a potential of $-0.25 \mathrm{~V}$ (SHE) and it is inhibited by the thin film electrode oxidation ( $0.1 \mathrm{~V}$ vs. SHE), which is indicated by a sudden drop of the current density value to approximately $2.01 \mathrm{~mA} \mathrm{~cm}^{-2}$. Increasing the Pd content in the thin film alloy caused a shift of the oxidation peak to a more negative potential $(0.09 \mathrm{~V}$ vs. SHE for $\mathrm{Cu}-21.9$ at.\% Pd). At more positive potential region, an additional anodic peak occurs with increasing Pd amount. At approximately $0.85 \mathrm{~V}$ (SHE) for the highest Pd concentration, the obtained anodic peak can be attributed to the $\mathrm{PdO}$ and $\mathrm{PdO}_{2}$ formation [34]. The maximum of the current density peak for the glucose oxidation peak in the anodic scan direction was plotted as a function of the Pd concentration in order to determine the electrocatalytic properties towards the glucose oxidation within the material library (Fig. 7). It can be clearly seen that with increasing the Pd content in the $\mathrm{Cu}-\mathrm{Pd}$ thin film combinatorial material library, an increased current density value was obtained. An optimum of the current density value was found for a Pd concentration of 8.2 at.\% at approximately $7.33 \mathrm{~mA} \mathrm{~cm}^{-2}$. Comparing the current density value of the starting composition (2.8 at.\% Pd) with the obtained current value for a Pd amount of 8.2 at.\% showing the highest catalytic effect, the latter is approximately 1.4 times higher. A further increase of the Pd concentration in the $\mathrm{Cu}-\mathrm{Pd}$ thin film alloys showed a decrease in the electrocatalytic effect. For a Pd content of 10.3 at.\%, the current density value retains $80 \%$ of the current density value for the highest catalytic effect. The current density values leveled off a constant plateau at approximately $4.5 \mathrm{~mA} \mathrm{~cm}^{-2}$ for higher Pd concentrations (> 11.7 at. $\%$ Pd). The compositional gradient of the $\mathrm{Cu}-\mathrm{Pd}$ thin film combinatorial library was chosen in this way that an overlapping part in the Pd concentration was obtained. As evidenced from Fig. 7, for adjacent samples, similar current density values were obtained independently from the sample number. For samples I and II, the maximum current density value was measured for a Pd content of 8.2 at.\%. These results lead to the conclusion that the Pd content plays a major role in the glucose oxidation process. Additionally, large variations in the local surface roughness were found in this compositional region $(7.5-10.5$ at.\% $\mathrm{Pd})$ (Fig. 3). Therefore, it is possible that the enhanced electrocatalytic properties occur due to a suitable Pd content combined with this local non-uniformity in the surface features which may trigger improved adsorption, desorption, and/or kinetic effects. For a similar Pd content (7.5 at.\%), enhanced properties for electrocatalytic oxidation of formaldehyde were also 


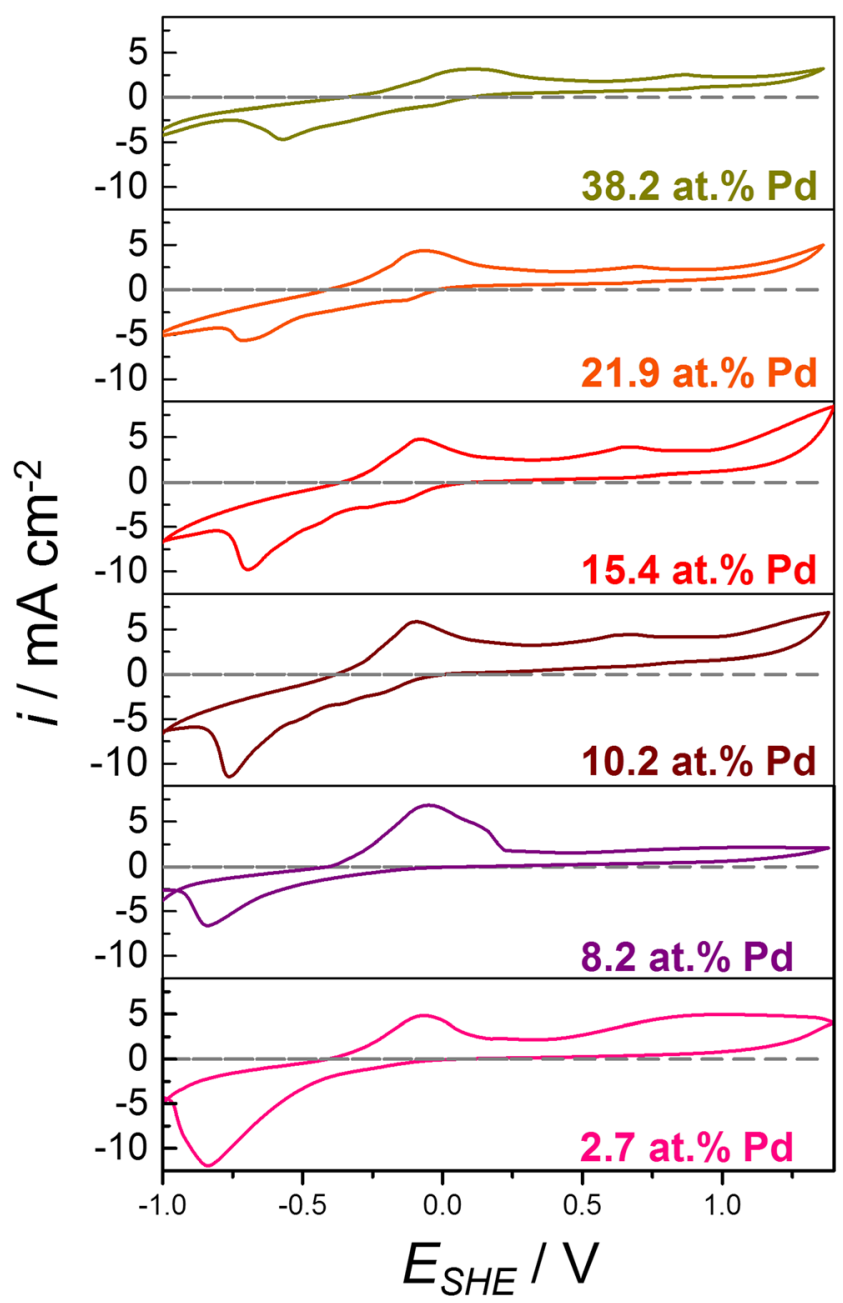

Fig. 6 Selected $\mathrm{CV}$ of $\mathrm{Cu}-\mathrm{Pd}$ thin film combinatorial library at various $\mathrm{Pd}$ concentrations with (continuous line) and without (dotted line) $35 \mathrm{mM}$ glucose found on $\mathrm{Cu}-\mathrm{Pd}$ sputtered combinatorial libraries and this feature was attributed to a combination between $\mathrm{Pd}$ concentration triggering different electron density on the surface, crystallite size, grain orientation, and surface microstructuring [9], facts which support as well the present findings.

Due to the easy fabrication as well as the good performance concerning the electrocatalytic oxidation of glucose, $\mathrm{Cu}-\mathrm{Pd}$ thin film alloys make this material a good candidate for being used as non-enzymatic glucose sensor and it can be further used for the glucose concentration detection. In order to investigate the glucose sensing efficiency, the amperometric response of the $\mathrm{Cu}-\mathrm{Pd}$ thin film combinatorial library was determined. Therefore, a time-dependent curve was recorded at the glucose oxidation potential $(-0.05 \mathrm{~V})$ for the $\mathrm{Cu}-8.2$ at.\% $\mathrm{Pd}$, which showed the highest electrocatalytic efficiency. Figure 8 presents the results of the amperometric response after the successful addition of various glucose concentrations (0-100 mM), which are indicated by an arrow. This experiment was performed under flowing conditions with a flow rate in the range of milliliters per hour that allows quasi-stationary conditions. The use of a flow cell conferred the possibility to investigate the online glucose monitoring behavior of the sensing material (e.g., environmental monitoring, blood glucose concentration $[11,36])$. The measurement without the addition of glucose is shown as background level. The current density reached a plateau at $0.15 \mu \mathrm{A} \mathrm{cm}^{-2}$ after $10 \mathrm{~s}$. The current density value increased after the addition of a welldefined amount of glucose, and the glucose oxidation process was indicated by a steady state current density plateau. From Fig. 8, it can also be observed that the increase of the current density plateau is based on an equidistant increment, which can be related to the glucose concentration. To determine the
Fig. 7 The maximum current density of the glucose oxidation peak as a function of the $\mathrm{Pd}$ concentration along the entire compositional spread

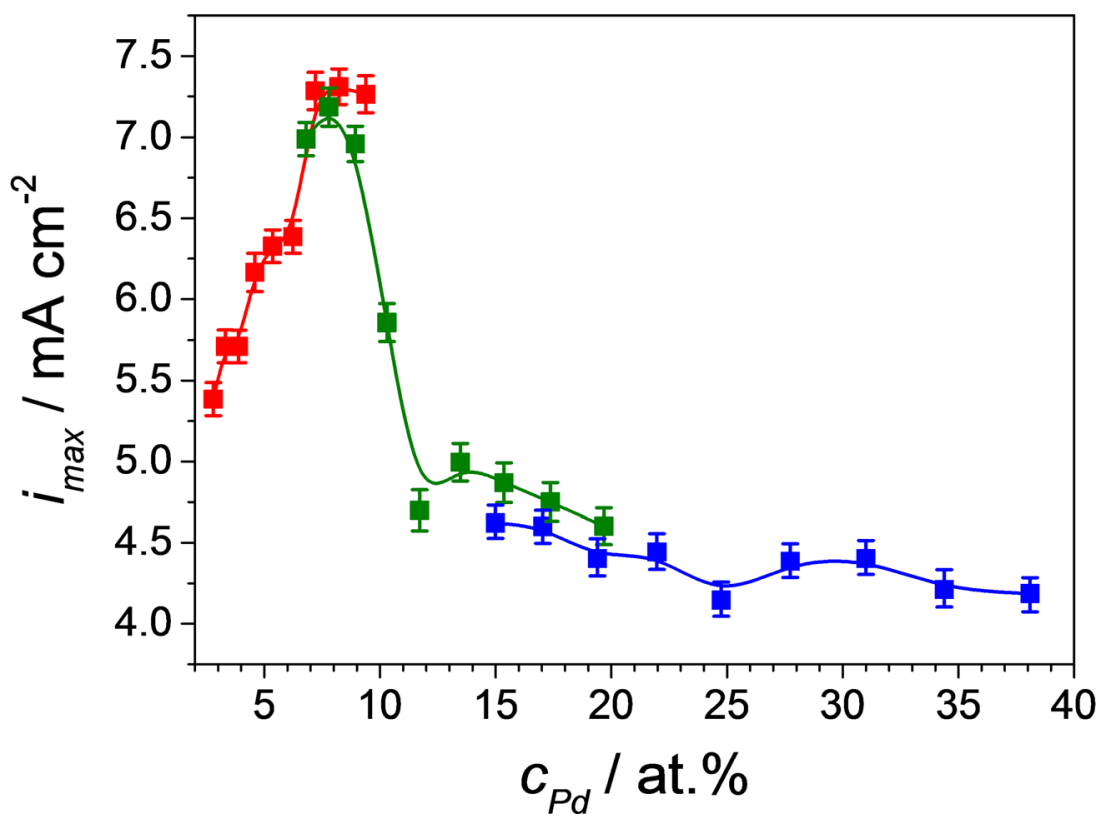


Fig. 8 Amperometric response after the addition of various glucose concentrations at the oxidation potential. The inlet presents the calibration curve for the glucose at the $\mathrm{Cu}-8.2$ at.\% $\mathrm{Pd}$

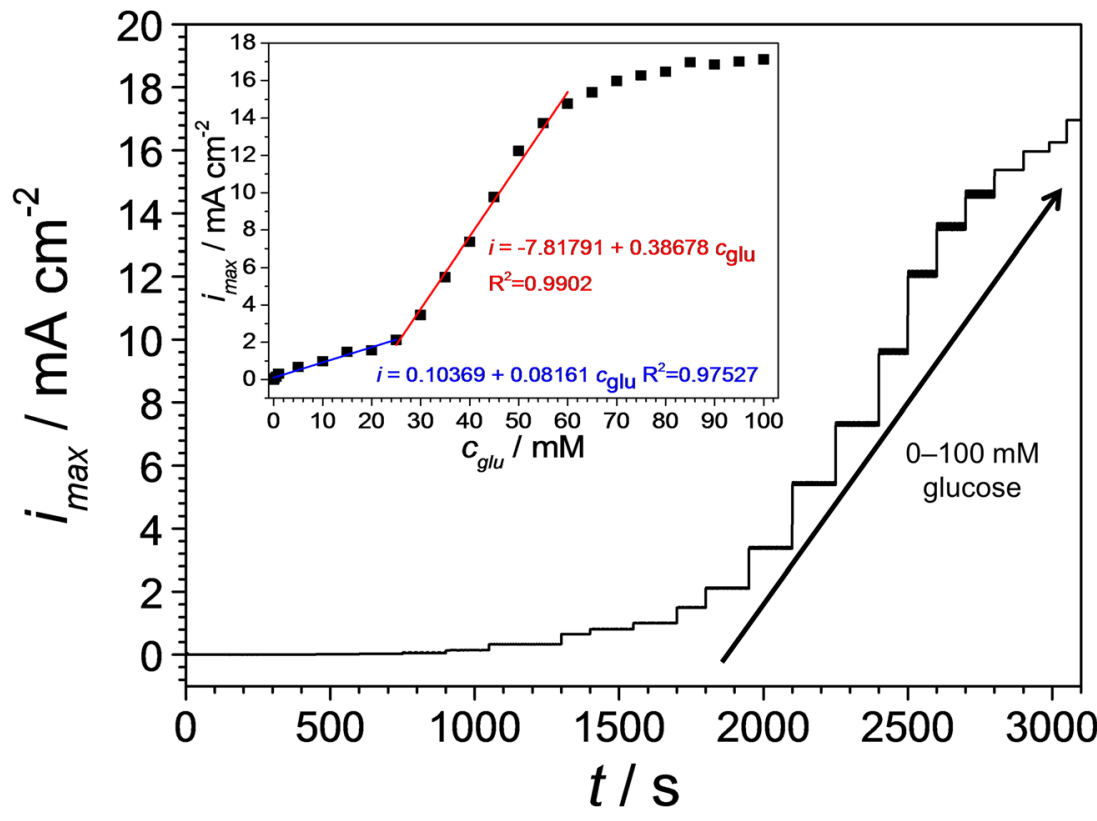

sensing efficiency of the $\mathrm{Cu}-8.2$ at.\% $\mathrm{Pd}$ thin film alloy the value of the current density plateau was plotted as function of the glucose concentration. The inlet of Fig. 8 depicts the calibration curve of the $\mathrm{Cu}-8.2$ at.\% $\mathrm{Pd}$ thin film alloy. A limiting current density saturation level was reached above $65 \mathrm{mM}$ glucose in the electrolyte solution. At higher glucose concentrations, the intermediate species adsorb on the $\mathrm{Cu}-\mathrm{Pd}$ surface, and consequently, more time is needed for fresh glucose molecules to reach the electrode surface, which is in good agreement with previous studies [37]. In the lower glucose concentration region $(0-60 \mathrm{mM})$, two linear regions were obtained. The first linear region is the range $0-25 \mathrm{mM}$ and the second one, from 25 to $60 \mathrm{mM}$ glucose. From the slope of the fitted calibration curve, an increase of the current density value of approximately $81.6 \mu \mathrm{A} \mathrm{cm}^{-2}$ is obtained after the addition of $1 \mathrm{mM}$ glucose electrolyte solution in the lower concentration range $(0-25 \mathrm{mM})$. In the higher concentration range $(25-$ $60 \mathrm{mM}$ ), the addition of $1 \mathrm{mM}$ glucose solution caused an increase of approximately $386 \mu \mathrm{A} \mathrm{cm}^{-2}$. Performing a linear fitting of the calibration data yields a correlation coefficient of 0.9752 (0-25 mM glucose) and 0.9902 (25-60 mM glucose). The observed linear range is in good agreement with previous studies. In Table 1, the results of these studies [38] are summarized.

Comparing the $\mathrm{Cu}-8.2$ at.\% $\mathrm{Pd}$ with various $\mathrm{Cu}$ and $\mathrm{Pd}$ alloys, as well as noble metal electrode materials, the investigated thin film alloys showed a wide linear concentration range. Additionally, the lower detectable concentration range of the current work is in the physiological level $(3-8 \mathrm{mM}$ glucose), and the upper detection limit is far above the physiological range. This aspect makes these particular alloys (approximately 7.5 to 10.5 at.\% $\mathrm{Pd}$ in $\mathrm{Cu}$ ) easily implementable in glucose detection devices for physiological levels [35].
Table 1 Comparison of the performance of several different, modified electrodes for non-enzymatic glucose oxidation

\begin{tabular}{|c|c|c|c|c|}
\hline Electrode & $\mathrm{pH}$ & Ep vs. SHE/V & $\begin{array}{l}\text { Linear } \\
\text { range/mM }\end{array}$ & $\begin{array}{l}\text { Detection } \\
\text { limit } / \mu \mathrm{M}\end{array}$ \\
\hline $\mathrm{Cu}-\mathrm{Pd}$ & 7.4 (PBS) & -0.05 & $0.005-60$ & 5 \\
\hline Pd-SWNTs $^{35}$ & 7.4 (PBS) & -0.05 & $0.5-17$ & 0.2 \\
\hline $\mathrm{Pt}-\mathrm{PbNA}{ }^{35}$ & 7.4 (PBS) & 0.1 & up to 11 & 8 \\
\hline $\mathrm{PtPb}-\mathrm{CNTs}^{35}$ & 7.4 (PBS) & 0.05 & up to 5 & 7 \\
\hline Porous gold ${ }^{35}$ & 7.4 (PBS) & 0.55 & $2-10$ & 5 \\
\hline nanoCu ${ }_{2} \mathrm{~S}-\mathrm{CNTs}{ }^{35}$ & $13.0(\mathrm{NaOH})$ & 0.7 & - & 5 \\
\hline $\mathrm{Pd}-\mathrm{Cu}=\mathrm{GCE}^{33}$ & 7.0 (PBS) & 0.81 & $0.7-100$ & 0.1 \\
\hline $\mathrm{Pd} / \mathrm{GE}^{38}$ & $13.0(\mathrm{NaOH})$ & 0.14 & $0.01-5$ & 1 \\
\hline $\mathrm{Pd}_{66} \mathrm{Cu}_{34} / \mathrm{GE}^{38}$ & $13.0(\mathrm{NaOH})$ & -0.15 & $1-18$ & 20 \\
\hline
\end{tabular}

SWNTS single-walled carbon nanotubes, NA nanowire array, $C N T s$ carbon nanotubes, GCE glassy carbon electrode, $G E$ graphene 
Even the addition of a small amount of Pd (8.2 at.\%) showed a high electrocatalytic effect towards glucose oxidation due to the surface adsorption modification. Furthermore, this good performance of the electrode material is due to the alloyed $\mathrm{Cu}$, which is responsible for a lowering effect of the electronic binding energy of Pd [33]. The fabricated Cu-Pd non-enzymatic glucose sensor exhibits a good sensitivity, low detection limit, and a wide linear concentration range compared to previously investigated glucose electrode materials. Additionally, important parameters such as reproducibility, repeatability, and stability for sensing materials have been tested. For these purposes, all electrochemical investigations were performed in a mixture of $35 \mathrm{mM}$ glucose and a PBS pH 7.4 using the FT-SDCM. First, the reproducibility of the fabricated Cu-Pd thin film combinatorial library was investigated for all thin film alloys along the entire compositional spread. Cyclic voltammetric measurements were recorded on the same library and substrate, and the results are presented in Fig. 7 (error bars). The relative standard deviation was not larger than 5\% for the investigated composition. Comparing the adjacent samples, which were fabricated under the same conditions, similar results of the observed current density values for identical $\mathrm{Cu}$-Pd compositions at the maximum of the glucose oxidation peaks were obtained. Furthermore, the stability of the $\mathrm{Cu}-10.2$ at.\% Pd thin film alloy was investigated by recording cyclic voltammograms under a constant flow with a flow rate in the range of milliliters per hour. All stability measurements were performed on the same spot, and the sensing material was rinsed with distilled water as well as dried with nitrogen gas. The $\mathrm{Cu}-\mathrm{Pd}$ thin film alloy was stored in a clean place under laboratory conditions. Figure 9 shows the recorded cyclic voltammograms after 7, 14, and 21 days. After
7 days, the current density value decreases with $3 \%$ and after 14 days with $6 \%$ from the initial current density value (approximately $6 \mathrm{~mA} \mathrm{~cm}^{-2}$ ). The obtained current density value at the maximum of the glucose oxidation peak remains $89 \%$ from the initial current density value after 21 days. Overall, the studied $\mathrm{Cu}-\mathrm{Pd}$ thin film alloy showed excellent reproducibility, repeatability, and stability as glucose sensor.

Since additional compounds such as ascorbic acid (AA) and uric acid (UA) are found in glucose solutions (e.g., blood), selectivity studies for glucose detections have been performed. For this purpose, a defined amount of AA and UA (20 mM each) was added to the PBS pH 7.4 electrolyte solution. In an attempt to study the influence or interference of these organic compounds, amperometric measurements were performed using the FT-SDCM in order to determine the selectivity of the $\mathrm{Cu}-8.2$ at.\% Pd alloy. The flow cell simulates the real sample analyses, where such non-enzymatic glucose sensing materials are being used for online monitoring of glucose concentration. In Fig. 10, the results of the amperometric detection of the selectivity study are presented. The measurements were performed with and without the addition of AA and UA solution at the glucose oxidation potential of $-0.05 \mathrm{~V}$ (SHE). After $100 \mathrm{~s}$, the current density reached a background level. First, the organic compounds were added to the electrolyte solution. The inlet of Fig. 10 depicts the result of the amperometric response. The addition of AA resulted in an interfering detection signal of approximately $0.05 \mathrm{~mA} \mathrm{~cm}$. The detection error was found to be $5 \%$. After $100 \mathrm{~s}$, UA was added to the solution, and the increase of the current density value was approximately $10 \%$ higher than after the addition of AA. Considering the measurement error in the $\pm 5 \%$ range, a clear distinction cannot be made between the current density
Fig. 9 Cyclic voltammetric measurements performed on the Cu-10.2 at.\% Pd alloy after 7, 14, and 21 days showing the small decrease in the current density maximum, therefore indicating the high stability

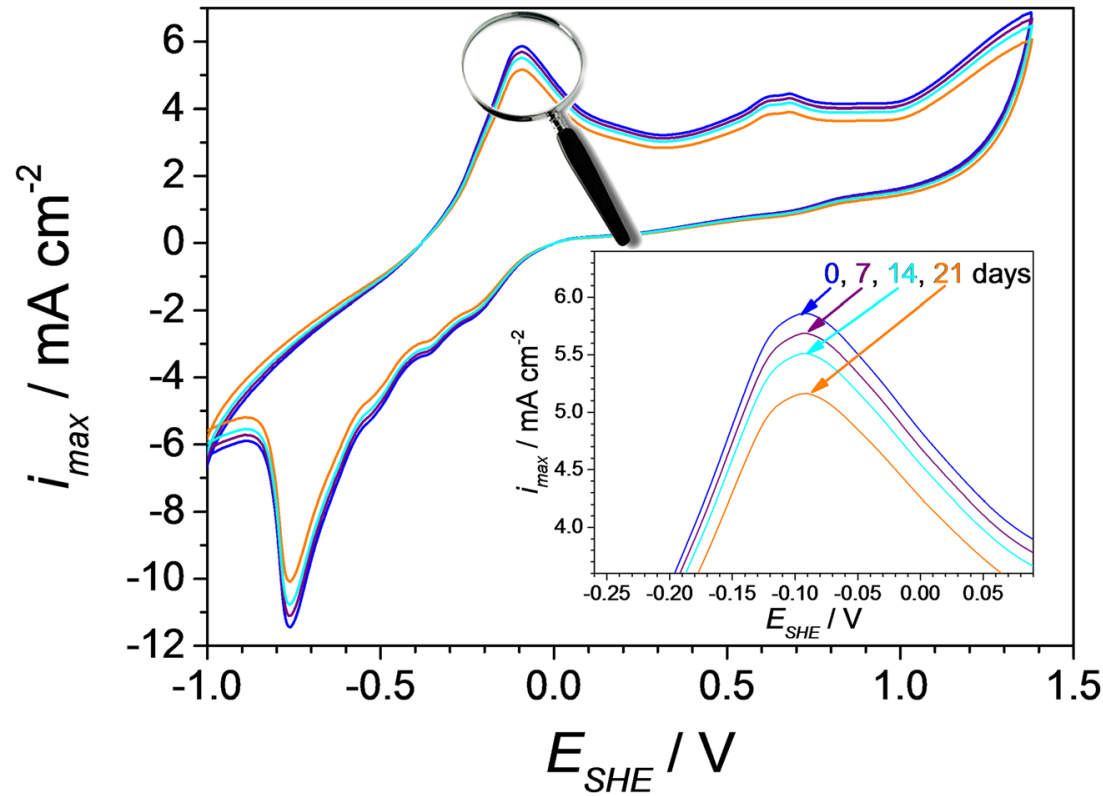


Fig. 10 Amperometric response of the $\mathrm{Cu}-8.2$ at.\% $\mathrm{Pd}$ in PBS $\mathrm{pH}$ 7.4 electrolyte solution with the addition of $10 \mathrm{mM}$ glucose, $20 \mathrm{mM}$ ascorbic acid (AA), and $20 \mathrm{mM}$ uric acid (UA)

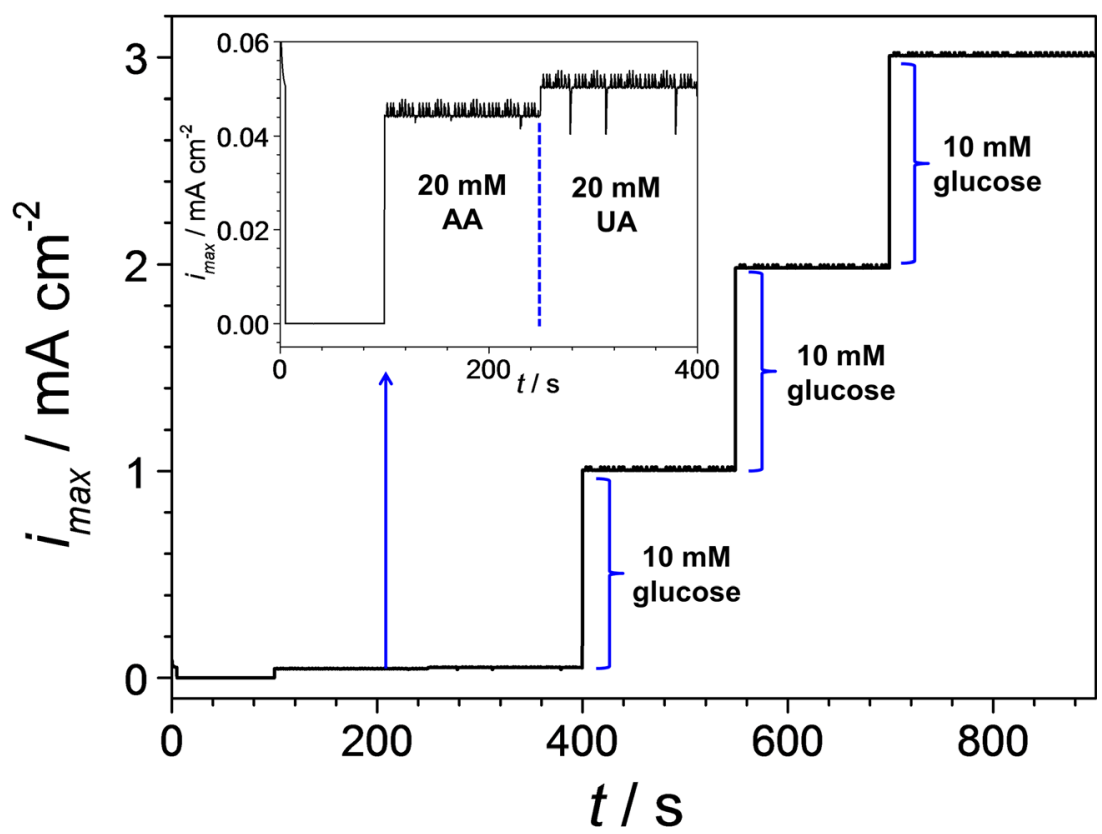

in the presence of AA as compared to AA + UA. The addition of $10 \mathrm{mM}$ glucose in the solution resulted in a very strong increase in the current density value, which confirms the previously observed results from the calibration curve. The glucose oxidation current density plateau was reached after $5 \mathrm{~s}$, which indicates a fast response. Additionally, there is a very clear distinguishability between the oxidation current density plateau and the background level due to the significant increase of approximately $1 \mathrm{~mA} \mathrm{~cm}{ }^{-2}$ after addition of $10 \mathrm{mM}$ glucose. Further addition of another $10 \mathrm{mM}$ glucose resulted in an increased current density plateau. Simultaneously, the equidistant increment of the current density value was observed which is in good agreement with the calibration curve (Fig. 8).

\section{Conclusions}

In the current work, the electrocatalytic oxidation of glucose was investigated on a $\mathrm{Cu}-\mathrm{Pd}$ thin film combinatorial library in a PBS pH 7.4 electrolyte solution. The Cu-Pd samples were fabricated by co-sputter deposition and they were screened along the entire compositional spread (2.6 at.\% to 39.2 at.\% Pd) using an FTSDCM. Cyclic voltammetric and chronoamperometric measurements were performed and the entire series of alloys showed suitability for being used in the glucose detection, but with different efficiencies. The highest electrocatalytic effect was found for a composition of 8.2 at.\% Pd. Additionally, the performance of this thin film alloy $(\mathrm{Cu}-8.2$ at.\% $\mathrm{Pd})$ towards glucose detection was tested. The sensor calibration performed in PBS solution containing different amounts of glucose revealed two linear regions with different sensitivities towards the quantitative glucose detection. The highest sensitivity was obtained in the range of 0-25 $\mathrm{mM}$ glucose in the electrolyte solution, which is indicated by an increase of $81.2 \mu \mathrm{A} \mathrm{cm}^{-2} \mathrm{mM}^{-1}$, normalized to the stepwise increase of $1 \mathrm{mM}$ glucose. Therefore, with the use of combinatorial thin film libraries, a very narrow compositional range of $\mathrm{Cu}$ alloys with $\mathrm{Pd}$ concentrations ranging from 7.5 to 10.5 at.\% was discovered, which demonstrates their suitability for being implemented in device fabrication as non-enzymatic glucose sensors with high amperometric response reproducibility (relative standard deviation 5\%), long-term stability, as well as selectivity to glucose oxidation.

Acknowledgements Open access funding provided by Johannes Kepler University Linz.

Funding The financial support by the Austrian Federal Ministry of Science, Research and Economy and the National Foundation for Research, Technology and Development through the Christian Doppler Laboratory for Combinatorial Oxide Chemistry (COMBOX) is gratefully acknowledged.

Open Access This article is distributed under the terms of the Creative Commons Attribution 4.0 International License (http:// creativecommons.org/licenses/by/4.0/), which permits unrestricted use, distribution, and reproduction in any medium, provided you give appropriate credit to the original author(s) and the source, provide a link to the Creative Commons license, and indicate if changes were made.

\section{References}

1. J.P. Kollender, A.I. Mardare, A.W. Hassel, Multi-scanning droplet cell microscopy (multi-SDCM) for truly parallel high throughput electrochemical experimentation. Electrochim. Acta 179, 32 (2015) 
2. S.O. Klemm, J.-C. Schauer, B. Schuhmacher, A.W. Hassel, High throughput electrochemical screening and dissolution monitoring of Mg-Zn material libraries. Electrochim. Acta 56, 9627 (2011)

3. T.H. Muster, A. Trinchi, T.A. Markley, D. Lau, P. Martin, A. Bradbury, A. Bendavid, S. Dligatch, A review of high throughput and combinatorial electrochemistry. Electrochim. Acta 56, 9679 (2011)

4. W. Burgstaller, M. Hafner, M. Voith, A.I. Mardare, A.W. Hassel, Copper-nickel oxide thin film library reactively co-sputtered from a metallic sectioned cathode. J. Mater. Res. 29, 148 (2014)

5. C.C. Mardare, A.W. Hassel, Investigations on bactericidal properties of molybdenum-tungsten oxides combinatorial thin film material libraries. ACS Comb. Sci. 16, 631 (2014)

6. M. Hafner, W. Burgstaller, A.I. Mardare, A.W. Hassel, Aluminiumcopper-nickel thin film compositional spread: Nickel influence on fundamental alloy properties and chemical stability of copper. Thin Solid Films 580, 36 (2015)

7. J.P. Kollender, A.I. Mardare, A.W. Hassel, Localized photoelectrochemistry on a tungsten oxide-iron oxide thin film material library. ACS Comb. Sci. 15, 601 (2013)

8. C.D. Grill, I. Pötzelberger, J.P. Kollender, A.W. Hassel, Cobaltnickel material libraries obtained from electrodeposition using citrate or glycine as additives. Phys. Status Solidi A 213, 1417 (2016)

9. I. Pötzelberger, C.C. Mardare, W. Burgstaller, A.W. Hassel, Maximum electrocatalytic oxidation performance for formaldehyde in a combinatorial copper-palladium thin film library. Appl. Catal. A Gen. 525, 110 (2016)

10. M. Keusgen, Biosensors: new approaches in drug discovery. Naturwissenschaften 89, 433 (2002)

11. J. Gun, M.J. Schöning, M.H. Abouzar, A. Poghossian, E. Katz, Field-effect nanoparticle-based glucose sensor on a chip: amplification effect of coimmobilized redox species. Electroanalysis 20, 1748 (2008)

12. R.K. Shervedani, M. Karevan, A. Amini, Prickly nickel nanowires grown on $\mathrm{Cu}$ substrate as a supersensitive enzyme-free electrochemical glucose sensor. Sensors Actuators B Chem. 204, 783 (2014)

13. J. Chen, J. Zheng, A highly sensitive non-enzymatic glucose sensor based on tremella-like $\mathrm{Ni}(\mathrm{OH})_{2}$ and $\mathrm{Au}$ nanohybrid films. J. Electroanal. Chem. 749, 83 (2015)

14. Y. Chen, W. Schuhmann, A.W. Hassel, Electrocatalysis on gold nanostructures: is the $\{110\}$ facet more active than the $\{111\}$ facet? Electrochem. Commun. 11, 2036 (2009)

15. X. Zhong, R. Yuan, Y. Chai, In situ spontaneous reduction synthesis of spherical Pd@ Cys- $\mathrm{C}_{60}$ nanoparticles and its application in nonenzymatic glucose biosensors. Chem. Commun. 48, 597 (2012)

16. M. Li, X. Bo, Y. Zhang, C. Han, L. Guo, One-pot ionic liquidassisted synthesis of highly dispersed PtPd nanoparticles/reduced graphene oxide composites for nonenzymatic glucose detection. Biosens. Bioelectron. 56, 223 (2014)

17. H.-F. Cui, J.-S. Ye, W.-D. Zhang, C.-M. Li, Luong, H.T. John, F.-S. Sheu, Selective and sensitive electrochemical detection of glucose in neutral solution using platinum-lead alloy nanoparticle/carbon nanotube nanocomposites. Anal. Chim. Acta 594, 175 (2007)

18. F. Xiao, F. Zhao, Y. Zhang, G. Guo, B. Zeng, Ultrasonic electrodeposition of gold-platinum alloy nanoparticles on ionic liquidchitosan composite film and their application in fabricating nonenzyme hydrogen peroxide sensors. J. Phys. Chem. C 113, 849 (2009)

19. F. Xiao, F. Zhao, D. Mei, Z. Mo, B. Zeng, Nonenzymatic glucose sensor based on ultrasonic-electrodeposition of bimetallic PtM (M
$=\mathrm{Ru}, \mathrm{Pd}$ and $\mathrm{Au}$ ) nanoparticles on carbon nanotubes-ionic liquid composite film. Biosens. Bioelectron. 24, 3481 (2009)

20. I. Pötzelberger, A.I. Mardare, A.W. Hassel, Electrocatalytic oxidation of glucose by screening combinatorial copper-nickel alloys. Phys. Status Solidi A 213, 1434 (2016)

21. I. Pötzelberger, A.I. Mardare, A.W. Hassel, Screening of catalytic effects on copper-zinc thin film combinatorial libraries for formaldehyde oxidation. Phys. Status Solidi A 212, 1184 (2015)

22. R. Ojani, J.-B. Raoof, Y. Ahmady-Khanghah, S. Safshekan, Copper-poly (2-aminodiphenylamine) composite as catalyst for electrocatalytic oxidation of formaldehyde in alkaline media. Int. J. Hydrog. Energy 38, 5457 (2013)

23. J.P. Kollender, M. Voith, S. Schneiderbauer, A.I. Mardare, A.W. Hassel, Highly customisable scanning droplet cell microscopes using 3D-printing. J. Electroanal. Chem. 740, 23 (2015)

24. K.A. Lill, A.W. Hassel, A combined $\mu$-mercury reference electrode/ Au counter-electrode system for microelectrochemical applications. J. Solid State Electrochem. 10, 941 (2006)

25. A.I. Mardare, A. Savan, A. Ludwig, A.D. Wieck, A.W. Hassel, A combinatorial passivation study of Ta-Ti alloys. Corros. Sci. 51, 1519 (2009)

26. A.I. Mardare, A.D. Wieck, A.W. Hassel, Microelectrochemical lithography: a method for direct writing of surface oxides. Electrochim. Acta 52, 7865 (2007)

27. A. J. Bard, R. Parson, J. Jordan (Ed.), Standard potentials in aqueous solution, INC, New York and Basel, 1985

28. A. Vaškelis, E. Norkus, I. Stalnionien, G. Stalnionis, Effect of the $\mathrm{Cu}$ electrode formation conditions and surface nano-scale roughness on formaldehyde anodic oxidation. Electrochim. Acta 49, 1613 (2004)

29. K.E. Toghill, R.G. Compton, Electrochemical non-enzymatic glucose sensors: a perspective and an evaluation. Int. J. Electrochem. Sci. 5, 1246 (2010)

30. M. Pasta, F. La Mantia, Y. Cui, Mechanism of glucose electrochemical oxidation on gold surface. Electrochim. Acta 55, 5561 (2010)

31. A. Brouzgou, P. Tsiakaras, Electrocatalysts for glucose electrooxidation reaction: a review. Top. Catal. 58, 1311 (2015)

32. M.H. Martin, A. Lasia, Study of the hydrogen absorption in Pd in alkaline solution. Electrochim. Acta 53, 6317 (2008)

33. X. Zhu, G. Kang, X. Lin, PdCu alloy nanoclusters: generation and activity tuning for electrocatalytic oxidation of nitrite. Microchim. Acta 159, 141 (2007)

34. M. Pourbaix (ed.), Atlas of Electrochemical Equilibria in Aqueous Solutions (NACE, Houston, 1974)

35. L. Meng, J. Jin, G. Yang, T. Lu, H. Zhang, C. Cai, Nonenzymatic electrochemical detection of glucose based on palladium-singlewalled carbon nanotube hybrid nanostructures. Anal. Chem. 81, $7271(2009)$

36. P. Lu, J. Yu, Y. Lei, S. Lu, C. Wang, D. Liu, Q. Guo, Synthesis and characterization of nickel oxide hollow spheres-reduced graphene oxide-nafion composite and its biosensing for glucose. Sens. Actuator B: Chem. 208, 90 (2015)

37. K.-C. Lin, Y.-C. Lin, S.-M. Chen, A highly sensitive nonenzymatic glucose sensor based on multi-walled carbon nanotubes decorated with nickel and copper nanoparticles. Electrochim. Acta 96, 164 (2013)

38. M. Yuan, A. Liu, M. Zhao, W. Dong, T. Zhao, J. Wang, W. Tang, Bimetallic $\mathrm{PdCu}$ nanoparticle decorated three-dimensional graphene hydrogel for non-enzymatic amperometric glucose sensor. Sensors Actuators B Chem. 190, 707 (2014) 